\title{
Surface wettability and energy effects on the biological performance of poly-3-hydroxybutyrate films treated with RF plasma
}

\author{
D.S. Syromotina, R.A. Surmenev, M.A. Surmeneva, A.N. Boyandin, E.D. Nikolaeva, O. \\ Prymak M. Epple, M. Ulbricht, C. Oehr, T.G. Volova
}

Abstract

The surface properties of poly-3-hydroxybutyrate (P3HB) membranes were modified using oxygen and an ammonia radio-frequency (RF, $13.56 \mathrm{MHz}$ ) plasma. The plasma treatment procedures used in the study only affected the surface properties, including surface topography, without inducing any significant changes in the crystalline structure of the polymer,with the exception being a power level of $250 \mathrm{~W}$. Thewettability of themodified P3HB surfaces was significantly increased after the plasma treatment, irrespective of the treatment procedure used. Itwas revealed that both surface chemistry and surface roughness changes caused by the plasma treatment affected surface wettability. A treatment-induced surface aging effect was observed and resulted in an increase in the water contact angle and a decrease in the surface free energy. However, the difference in the water contact angle between the polymers that had been treated for 4 weeks and the untreated polymer surfaces was still significant. A dependence between cell adhesion and proliferation and the polar component of the surface energy was revealed. The increase in the polar component after the ammonia plasma modification significantly increased cell adhesion and proliferation on biodegradable polymer surfaces compared to the untreated P3HB and the P3HB modified using an oxygen plasma.

\section{Introduction}

Themost prospective routes for fabricating newmaterials for artificial organs and tissues that are capable of biodegrading in the body, i.e., they are gradually degraded and replaced by an individual's own cells and tissues, are being investigated. Therefore, biodegradable polymers are of great interest, particularly for the fabrication of biodegradable bone implants [1,2]. Among all known biodegradable materials, the polyesters of hydroxycarboxylic acids, i.e., polyhydroxyalkanoates (PHAs), are the most prospective class of materials that are being studied for diverse biomedical applications. These polymers possess good biocompatibility as well as physical and mechanical properties that are similar to that of the hard tissue in the human body, making them promising materials for use in reconstructing bone tissue. Despite all of the advantages of PHAs, they have not yet gained a wide clinical use due to the hydrophobic nature of their surface which prevent cell adhesion and growth. Among PHAs a homopolyester of 3hydroxybutyric acid, poly-3-hydroxybutyrate (P3HB), is themost studied polymer. It is thermoplastic and has good strength characteristics in comparison with widely used polylactides. $\mathrm{P} 3 \mathrm{HB}$ is slowly biodegraded in an organism producing non-toxic 3-hydroxybutyric acid and, therefore, it can be used as amaterial for a wide spectrum of biomedical applications - from sutures and drug delivery systems to matrices for tissue engineering and reconstructive surgery [3-6]. However, high degree of crystallinity of P3HB (over 70\%) leads to a high rigidity and brittleness of its products, and their low resistance to stretching [7,8]. Moreover, bioresorption rate of this polymer is low (from several months to a few years) that can be undesirablewhen healing of the damaged tissue occurs quicker inducing adverse foreign body reaction [9]. The lack of mechanical stability during degradation can also occur. As other PHAs, P3HB has highly enough hydrophobicity, that, in some cases, can prevent normal interaction of polymer surface with biological media of the organism and also cell adhesion [10-13]. However, the wettability of the P3HB may vary with the structure and composition of the surface, e.g., the surface topography, content of the crystalline and amorphous phases, and the presence of polar functional groups with a specific charge [10]. Radio-frequency (RF) plasma is usually used to treat the surface of different polymers $[14,15]$. Reactive gases allowus to obtain the necessary functional groups on the P3HB surface and also impart other properties, such as bioactivity [16- 
19]. The data on the influence of the different working parameters of plasma, such as the type of gas, power, or pressure, on the changes in the properties of PHA, particularly hydrophilicity, is limited. The majority of the studies did not investigate the changes in the surface wettability over time. It is known that the surface aging effect can play a significant role, due to the effect of the atmosphere on the surface properties of polymers. In one study [17], the hydrophilic properties of the plasma-treated biodegradable polymers deteriorated over time. Thus, the objective of the present study was to modify thin films of P3HB with a low-temperature radio-frequency (RF) plasma using reactive gases (oxygen and ammonia) to improve the hydrophilic properties of the films by forming polar functional groups.

2. Materials andmethods

2.1. Preparation of the P3HB films P3HB films with a Mw of $959 \mathrm{kDa}$ and polydispersity of $Đ$ of 5.15 were obtained from the Institute of Biophysics of the Siberian Branch of the Russian Academy of Sciences (Russia, Krasnoyarsk) and produced by microbial biosynthesis using the B-10646 Cupriavidus eutrophus strain (this strain is registered in the Russian National Collection of Industrial Microorganisms). The bacteria were cultivated in sterile conditions in a BioFlo 115 cultivator ("New Brunswick", USA) in a volume of $7.5 \mathrm{~L}$ using a mineral medium containing glucose [20]. The properties of the polymer have been reported elsewhere [21-25]. Thin films of $\mathrm{P} 3 \mathrm{HB}$ were formed by casting a solution of the polymer in chloroform. Forty milliliters of the $2 \%$ polymer solution was heated to $35{ }^{\circ} \mathrm{C}$ and poured into clean Petri dishes. The films were allowed to dry for 3-5 days under normal conditions in a laminar flow hood. As a result, thin films were obtained in the form of $70 \mu \mathrm{m}$ thick disks that were approximately 10 $\mathrm{cm}$ in diameter.

\subsection{Plasma treatment of the P3HB films}

The P3HB films were treated with a low temperature plasma using a custom-made RF power generator operating at a frequency of $13.56 \mathrm{MHz}$ and oxygen or ammonia $(\mathrm{NH} 3)$ as the gaseous precursors $(99.99 \%)$. The treatment time was set at $20 \mathrm{~min}$ for all of the experiments. The oxygen plasma treatment was performed using RF power levels of $50 \mathrm{~W}, 100 \mathrm{~W}, 150 \mathrm{~W}$, $200 \mathrm{Wand} 250 \mathrm{Wat}$ a pressure of $30 \mathrm{~Pa}$ and a gas flow of 60 standard $\mathrm{cm} 3 \mathrm{~min}-1$. At the RF power of $100 \mathrm{~W}$, the pressure was set at 30,45 and $55 \mathrm{~Pa}$ for a gas flow 60,100 and 140 standard $\mathrm{cm} 3 \cdot \mathrm{min}-1$, respectively. The ammonia plasma treatment was performed at RF power levels of $50 \mathrm{~W}, 100 \mathrm{~W}, 150 \mathrm{~W}, 200 \mathrm{~W}$ and $250 \mathrm{~W}$ under a pressure of $30 \mathrm{~Pa}$ and a gas flow of 30 standard $\mathrm{cm} 3 \mathrm{~min}-1$.

2.3. Investigation of the surface topography

The surface topography of the treated and untreated polymers was investigatedwith an ESEMQuanta 400 FEG scanning electronmicroscope operated at $15 \mathrm{kV}$. The surface roughness was investigated using atomic force microscope (AFM, SOLVER) (NT-MDT, Russia) in noncontact mode. Three roughness parameters were considered to evaluate the surface characteristics of the polymers: average roughness $(\mathrm{Sa})$, root mean square $(\mathrm{Sq})$ and surface area ratio between the interfacial area and projected area (Sdr).

2.4. Investigation of the polymer structure

The phase composition and crystallinity of P3HB were analyzed by X-ray diffraction analysis (Bruker D8 ADVANCE, Germany) with $\mathrm{Cu} \mathrm{K} \alpha$ radiation in the $2 \theta$ range from 5 to $90^{\circ}$.

2.5. Fourier transform infrared (FT-IR) spectroscopy

Themolecular bonds of the sampleswere studied using an EQUINOX 55 (Bruker, Germany) in attenuated total reflectance (ATR) mode. The

infrared spectra of the P3HB films were obtained in the frequency range from 600 to $4000 \mathrm{~cm}-1$.

2.6. Water contact angle measurement

The contact angle analyses were performed with an optical contact angle apparatus (OCA 15 Plus Data Physics Instruments GmbH, Germany), using the SCA20 software (Data Physics Instruments $\mathrm{GmbH}$, Germany). The contact angle of water in air was measured by the sessile drop method. The entire analysis was conducted at $20 \pm 1{ }^{\circ} \mathrm{C}$. A minimum of 10 droplets $(2 \mu \mathrm{L}$, 
$5 \mu \mathrm{L} \cdot \mathrm{s}-1)$ of water and 5 droplets of diiodomethane or ethylene glycol were examined for each sample, and the resulting mean $\theta$ values were then used for the calculations. The surface free energy was calculated using the Owens-Wendt-Rabel-Kaelble (OWRK) method. Three different media (water, diiodomethane and ethylene glycol)were used for these calculations. The theory of surfacewetting, aswell as contact angle hysteresis is described inmore detail in Ref. [16-18].

\subsection{Cell proliferation study}

To estimate the adhesion properties of the studied samples, disks with the diameter of 15 $\mathrm{mm}$ were prepared. The disks were sterilized using $70 \%$ ethanol for $10 \mathrm{~min}$ followed by subsequent UV-irradiation. Then, they were placed in 24-alveolar plates (TPP, Switzerland) and seeded with $104 \mathrm{NIH} 3 \mathrm{~T} 3$ mouse fibroblasts per $\mathrm{ml}$ of media. The fibroblastswere cultivated in Dulbecco'sModified EagleMedium (DMEM) with 10\% fetal calf serum and an antibiotics solution (streptomycin $100 \mu \mathrm{g} / \mathrm{ml}$, penicillin 100 units $/ \mathrm{ml}$ (Gibco, Invitrogen)) in a $37{ }^{\circ} \mathrm{C}$ incubator in a 5\% CO2 atmosphere. The media was changed once every three days. The adhesion properties of the polymer surfaces were compared by taking into account the results for cell attachment, cell number and morphology based on the SEM images (TM 3000, Hitachi, Japan), as well as the fluorescence staining of the cells using DAPI (DNA marker) and FITC (amarker of the actin proteins in the cytoplasm). After fixation, the cells were washed three times in Dulbecco's Phosphate-Buffered Saline (DPBS) and incubated in DAPI solution for 5 min, followed by washing in DPBS. The optical density was determined using an iMark spectrophotometer (Bio-Rad, USA) after dissolving the MTT-formazane crystals in DMSO.

3. Results and discussion

3.1. Surface morphology

It is known that the plasma treatment affects the polymer surface and that the following processes can occur: heating, breaking of chemical bonds, degradation, etc. [26]. Allof theseprocessesmay significantly change the structure and morphology of the polymers. The SEM images of the surface and structural changes in the P3HB films before and after the plasma treatment and their cross-sectional profiles are presented in Fig. 1. The plasma treatment changed the relief of the polymer surface. The surface of the untreated P3HB is homogeneous and surface porosity is observed (Fig. 1b). After the oxygen plasma treatment, surface grooves ranging from 2 to $5 \mu \mathrm{m}$ (Fig. 1c) were formed. Moreover, surface porosity was not observed. The roughness parameters measured via AFM revealed that the increased RF power increased the surface roughness parameters to $\mathrm{Sa}=171 \pm 12 \mathrm{~nm}, \mathrm{Sq}=217 \pm 18 \mathrm{~nm}, \mathrm{Sdr}=22.3 \%$ for the oxygen plasma and $\mathrm{Sa}=181 \pm 12 \mathrm{~nm}, \mathrm{Sq}=229 \pm 14 \mathrm{~nm}, \mathrm{Sdr}=63.5 \%$ for the ammonia plasma compared to the untreated P3HB $(\mathrm{Sa}=139 \pm 8 \mathrm{~nm}, \mathrm{Sq}=174 \pm 10 \mathrm{~nm}, \mathrm{Sdr}=16.2 \%)$. At a power level of $250 \mathrm{~W}$, the ammonia plasma had a larger impact on the morphology of the P3HB films than the oxygen plasma. Typical SEM images (Fig. 1e-f) demonstrated that an increase in the power to $250 \mathrm{~W}$ led to the destruction of the surface layers of the ammonia plasma-treated P3HB. It could be caused by hydrogen, which acts as a chemical agent and etches oxygen from the surface $[17,27]$.

\subsection{XRD analysis}

The XRD patterns of the untreated and plasma-modified P3HB surfaces are presented in Fig. 2. The results showed that $\mathrm{P} 3 \mathrm{HB}$ was a polycrystalline polymer. The plasma treatment procedure had no effect on the structure of the polymers, with the exception of the RF power of $250 \mathrm{~W}$. The XRD patterns showed peaks at $2 \theta$ of $13.5^{\circ}, 16.2^{\circ}, 20.2^{\circ}, 22.7^{\circ}, 25.6^{\circ}$ and $27.4^{\circ}$, which were in good agreement with the values reported for P3HB [28,29]. According to Scherrer's equation, the crystallite sizes of P3HB were ranged from 8 to $10 \mathrm{~nm}$. The polymers treated at $250 \mathrm{~W}$ revealed structural changes in the polymers modified using both working gas atmospheres. Variations in the parameters of the crystal unit cells were observed. At a power of $250 \mathrm{~W}$, the modified P3HB polymers had a smaller crystal unit cell by volume compared to the other cases (untreated P3HB: $\mathrm{a}=5.77 \mathrm{~A}, \mathrm{~b}=13.04 \mathrm{~A}, \mathrm{c}=6.08 \mathrm{~A}, \mathrm{~V}=458 \mathrm{~A} 3$; modified $\mathrm{P} 3 \mathrm{HB}$ via $\mathrm{O} 2$-plasma: $\mathrm{a}=5.69 \mathrm{~A}, \mathrm{~b}$ $=12.80 \mathrm{~A}, \mathrm{c}=5.98 \mathrm{~A}, \mathrm{~V}=437 \mathrm{~A} 3$; modified $\mathrm{P} 3 \mathrm{HB}$ via changes in the crystalline structure of 
P3HB after plasma treatment at a power of $250 \mathrm{~W}$ can be explained by considering the melting of the surface layers of the polymers.

\subsection{Chemical analysis}

The IR spectra of the untreated and modified P3HB are presented in Fig. 3. New peaks are absent after the plasma modification, indicating the absence of the formation of new bonds. The analysis indicated the presence of peaks with the following molecular groups: $\mathrm{C}-\mathrm{H}(2978 \mathrm{~cm}-1)$ and C_O $(1719 \mathrm{~cm}-1)$. Moreover, multiple bands were present in the range from $890 \mathrm{~cm}-1$ to $1453 \mathrm{~cm}-1$ (Fig. 3) [30,31]. Characteristic amino-related bonds, such as $\mathrm{C}-\mathrm{N}$ stretching at 1190 $1130 \mathrm{~cm}^{-1}$ or $\mathrm{N}-\mathrm{H}\left(1400-1000 \mathrm{~cm}^{-1}\right)$, were not identified. However, the amino $(\mathrm{C}-\mathrm{N})$ and amide ( $\left.\mathrm{N}-\mathrm{C} \_\mathrm{O}\right)$ groups on the polymer surface after the ammonia-plasma treatment were identified using X-ray photoelectron spectroscopy [15,17]. Therefore, the FT-IR ATR technique did not provide a clear understanding of the formation of amino, $\mathrm{C}-\mathrm{O}, \mathrm{C}-\mathrm{O}-\mathrm{C}$ or other groups because only the surface layers of the polymers were modified by the plasma and the information depth of the analysis was in the range of 1-2 $\mu \mathrm{m}$. An energy dispersive X-ray (EDX) analysis was performed to reveal the presence of the amino groups on the ammonia plasma-treated surface. The elemental composition of the polymers surface showed that the P3HB films primarily consisted of oxygen and carbon, and the ratio of carbon to oxygen was 3.03:1 for untreated P3HB. The plasma treatment only changed the atomic ratio of carbon to oxygen, which decreased after the oxygen plasma treatment (2.74:1). This ratio decreased even further with increasing oxygen pressure (2.62:1). This might indicate the formation of additional oxygencontaining bonds in the surface layers. The ammonia plasma treatment also decreased the $\mathrm{C} / \mathrm{O}$ ratio to 2.82:1. A similar trend in the $\mathrm{C} / \mathrm{O}$ ratiowas noted elsewhere [18]. The presence of nitrogen was detected in the surface layers of P3HB by EDS; however, the absolute amount could not be derived.

\subsection{Surface wettability}

Changes in thewettability of the P3HB polymers were observed after both the oxygen and ammonia plasma treatments. The measurements were performed immediately after the treatment and then every week within a 1 month period. Fig. 4a shows the effect of the plasma power on the water contact angle of the oxygen and ammonia plasmatreated P3HB films. Both gases caused a significant decrease in the static water contact angle compared to the untreated polymer $\left(85^{\circ} \pm 2^{\circ}\right)$. The plasma treatment at $50 \mathrm{~W}$ resulted in a decrease in the water contact angle. The lowest water contact angles were obtained at the RF power levels of 100 and $150 \mathrm{~W}$. An increase in the power to $200 \mathrm{~W}$ and $250 \mathrm{~W}$ increased the surface roughness, changed the structure of the polymers and, thus, deteriorated the surface wettability. It should also be noted that thewater contact angle of the polymers treated in ammonia plasma is significantly decreased compared to the polymers treated in oxygen plasma. These changeswere induced by the differences in the chemical composition of the surfaces. The calculations of the surface energy revealed that both of the plasma treatments significantly increased the surface energy (Fig. 5a). Importantly, the increase in the surface energy was mainly due to the increase in its polar component (the polar component of untreated $\mathrm{P} 3 \mathrm{HB}$ is $3.3 \mathrm{mN} / \mathrm{m}$ ). Furthermore, the lowest value of the water contact angle was achieved at the largest values of the polar component of the surface energy (note that the latter had been estimated based on the contact angle measurements in three different liquids). It is known that the surface of the polymers is exposed to the so-called "aging" process. This is a complex chemical and physical phenomenon that takes place on the polymer surface and is influenced by the environment during processing, use or storage. One prominent effect is the surface rearrangement of the functional segments to minimize the interfacial energy. As a result, the polymers' properties change. It is reported that the plasma-treated polymers'wettability deteriorates over time [17,32]. Figs. $4 \mathrm{~b}$ and5b showthe changes in thewater contact angle and surface energy within a four weeks period. As a result of aging, the water contact angle tends to increase. Although there were significant differences in the values of the contact angles between the ammonia and oxygen plasma-treated polymers after a 4weeks interval, the water contact angles were essentially equal. It was also observed that the increase in the contact angle is 
connected to the decrease in the polar component of the surface energy. Based on the results obtained in this study, the samples treated in oxygen plasma are less susceptible to the changes in the aging process compared to the samples treated in ammonia. Furthermore, it can be concluded that the surface of the modified polymers remains hydrophilic (contact angle is in the range of $35^{\circ}-40^{\circ}$ ) within a 1 month period. We also investigated the effect of the gas pressure in the vacuum chamber on thewettability of the P3HB surface. In this case, the plasma treatment was performed in oxygen atmosphere at a power of $100 \mathrm{~W}$. The choice of the power was based on our preliminary results (Fig. 4a) because the lowest value of the contact angle was obtained at a plasma power of $100 \mathrm{~W}$. Fig. 6 shows that the increase in the gas flow and pressure in the chamber decreased the water contact angle. This likely due to the increase in the number of particles bombarding the polymer and, therefore, a higher number of polar groups was formed. This was confirmed by the increase in the surface energy, including its polar component up to $39.5 \pm 1.0 \mathrm{mN} \cdot \mathrm{m}-1$, compared to that of the untreated polymers, which was $3.3 \pm 0.8 \mathrm{mN} \cdot \mathrm{m}-1$ (Fig. 7). At a pressure of $55 \mathrm{~Pa}$, the polar component of the surface energy exceeded that of the dispersive component. This explains the significant decrease in the contact angle of up to $16^{\circ} \pm$ $2^{\circ}$. The IR spectra did not reveal the formation of any new functional groups on the polymer surface with the increase in pressure, as shown in Fig. 3; therefore, it can be assumed that the surface energy changes can be explained by the increase in the density of the polar groups on the surface. The contact angle increased over time, as discussed above. The results obtained after plasma treatment at the pressures of 45 and $55 \mathrm{~Pa}$ differed from those measured at 1 week. The contact angle increased over time, even exceeding those obtained at $30 \mathrm{~Pa}$. However, there was no significant difference in the water contact angle between the pressure groups at 1 week after the surface treatment. The surface energy also significantly decreased at 1 week and did not significantly change within next 3 weeks. Although the water contact angle increased and the surface free energy decreased at 4 weeks after plasma treatment, they were still significantly lower compared to those observed for the untreated substrates. The roughness parameters measured via AFM revealed that the different RF power level resulted in the changes of the surface roughness parameters. Using the following equation, i.e. $\mathrm{r}=1+\mathrm{Sdr} / 100$, it was possible to obtain the values of the roughness factor (r). The followingWenzel equation $\cos \theta \mathrm{m}=\mathrm{r} \cos \theta \mathrm{y}(\theta \mathrm{m}$ - measured contact angle, $\theta \mathrm{y}$ - Young contact angle $)$ was used to derive the values of the Young contact angles using themeasured values of the contact angles. It is important to notice that theWenzel equation is based on the assumption that the liquid penetrates into the roughness grooves (Table 1) [33,34]. Taking into account the calculated values of the Young contact angles it is possible to conclude that both surface chemistry and surface roughness changes caused by the plasma treatment played a significant role in the surface wettability change of the treated polymers. Moreover, more pronounced effect of the surface roughness on the surfacewettabilitywas observed in the case of ammonia plasma treatment.

3.5. Cell proliferation study

Based on the wettability results (Fig. 4a), the modes of plasma treatment that achieved better wettability were chosen. Thus, the P3HB membranes were subjected to plasma treatment in oxygen and ammonia atmospheres at power levels of $100 \mathrm{~W}$ and $150 \mathrm{~W}$, a gas pressure of $30 \mathrm{~Pa}$ and a 20 min treatment timefor the further cultivation of NIH 3T3 fibroblasts. The plasma treatment affected the attachment and proliferative potential of fibroblasts cultured on the $\mathrm{P} 3 \mathrm{HB}$ films. Fig. 8 compares the cell density for the untreated and different plasma-treated samples after 7 days in culture. Evidently, not all of the plasma-modified membranes had a higher cell density than the untreated P3HB. The number of active and physiologically viable fibroblasts was calculated using DAPI staining, and the highest value was obtained for the cells cultured on the ammonia plasma-treated films $(3.5 \pm 0.3 .105 / \mathrm{cm} 2)$ compared to the control $(2.1 \pm 0.2$. $105 / \mathrm{cm} 2)$. SEMrevealed that the cell concentration for the untreated samples was $2.4 \pm 0.3$. $105 / \mathrm{cm} 2$ and that of the ammonia plasma-treated samples was $3.4 \pm 0.3 .105 / \mathrm{cm} 2$. The MTT test revealed increased metabolic activity of the fibroblasts on the ammonia plasma-treated surfaces compared to the untreated and oxygen plasma-treated films (Fig. 9). A statistical 
analysis of the influence of theNH3-plasma (150W) in theMTT test using the t-test showed the reliability of this effect at a significance level of 0.1 (tcrit. 1.533 and ttrial 1.939). The vitality of the cells on themodified polymers may be influenced by the surface topography, surface wettability, and surface chemical composition. It was found that the wettability of the polymers treated in the ammonia plasma was better than those treated in the oxygen plasma at power of $150 \mathrm{~W}$ measured 3 weeks after the plasma treatment (water contact angle: $50^{\circ} \pm 2^{\circ}$ for $\mathrm{O} 2$ modified $\mathrm{P} 3 \mathrm{HB}$ and $36^{\circ} \pm 2^{\circ}$ for NH3-modified P3HB), but their surface energy was essentially equal $(53.5 \pm 0.9 \mathrm{mN} / \mathrm{m}$ for O2-modified P3HB and $57.4 \pm 0.9 \mathrm{mN} / \mathrm{m}$ for NH3-modified P3HB). The difference is that the polar component of the NH3-modified P3HB is higher $(27.5 \mathrm{mN} / \mathrm{m})$ than that of the O2-modified polymers $(18.5 \mathrm{mN} / \mathrm{m})$. Other authors also reported that a higher value for the polar component of the surface energy led to a significant increase in cell viability and proliferation on plasma-treated surfaces [35]. It was also revealed that an increase in the polar component of the surface energy may improve osteoblast attachment and, thereby, osteoconduction [36]. It is necessary to note that even untreated P3HB is a non-toxic material which also shows no inflammatory reaction because hydrolytic degradation of P3HB results in the formation of 3-hydroxybutyric acid, a normal blood constituent [37,38]. Moreover, it was shown that P3HB in small concentrations $(0.02 \mathrm{~g}$. ml-1) can promote proliferation of L929 cells plated depending on cell density, and also significantly inhibit cell death [39]. The choice of P3HB for constructing biomedical devices can be stipulated for itsmechanical properties and surface hydrophobicity $[12,13]$. The intrinsic surface hydrophobicity of $\mathrm{P} 3 \mathrm{HB}$ can be overcome using various physical-chemical treatments including low temperature RF plasma. In this case, the treatment conditions affect only very thin polymer surface layers and preserve bulk structure, which allows to significantly change surface characteristics of the polymers without any significant changes of their bulk properties.

\section{Conclusions}

The influence of various oxygen and ammonia plasma treatment procedures on the properties of biodegradable $\mathrm{P} 3 \mathrm{HB}$ polymers was studied. The plasma treatment resulted in topographical changes compared to the initial substrates. It was revealed that both surface chemistry and surface roughness changes caused by the plasma treatment affected surface wettability. Hydrophilic surface behavior was revealed without any significant changes in the polymer composition with the exception of plasma treatment at a power level of $250 \mathrm{~W}$. A significant increase in the polar component of the surface energy was observed for both the oxygen and ammonia plasma treatments. Within 4 weeks after plasma exposure, the contact angle of the P3HB surfaces was significantly decreased compared to that of the untreated polymers; in addition, the surface energy of the plasma-treated surfaces was significantly, regardless of the gas atmosphere used. Furthermore, only the ammonia plasma-modified P3HB membranes improved the adhesion and proliferation of cells compared to the untreated and oxygen plasmatreated P3HB.

Acknowledgments

This research was supported by the State order NAUKA \#11.1359.2014/K, \#14.587.21.0013 (plasma treatment of the polymers) and the State budget allocated to the fundamental research at the Russian Academy of Sciences (project no 01201351505).

Fig. 1. The morphology of the P3HB polymers: (a) untreated P3HB, (b) cross-section of (a); (c) oxygen plasma-modified P3HB, $250 \mathrm{~W}$, (d) cross-section of (c); (e) ammonia plasma-modified P3HB, 250W, (f) cross-section of (e).

Fig. 2. XRD patterns of the untreated, oxygen plasma-modified and ammonia plasmamodified P3HB at different RF power levels.

Fig. 3. IR spectra of the untreated P3HB and the films treated with the different working gases at a power of $100 \mathrm{~W}$.

Fig. 4. The water contact angle: (a) as a function of the plasma power using the differentworking gases. The measurements were performed immediately after treatment; (b) obtained for the 
P3HB surfaces treated at $100 \mathrm{~W}$. The measurements were performed within 4 weeks after treatment: - - oxygen plasma; • — ammonia plasma.

Fig. 5. Surface energy of the P3HB polymers: (a) treated at the different power levels. The measurements were performed immediately after treatment; (b) treated at $100 \mathrm{~W}$. The measurements were performed within 4 weeks after treatment. Unshaded area — oxygen plasma; shaded area - ammonia plasma.

Fig. 6. The water contact angle as a function of the oxygen pressure at a power of $100 \mathrm{~W}$ : (a) the measurements were performed immediately after treatment; (b) the measurements were performed within a 1 month period after treatment: $\mathbf{-}-30 \mathrm{~Pa} ; \bullet-45 \mathrm{~Pa} ; \boldsymbol{\Delta}-55 \mathrm{~Pa}$.

Fig. 7. The surface energy of the oxygen plasma-treated P3HB at different gas pressures: (a) the measurements were performed after treatment; (b) the measurements were performed after treatment. Unshaded area - gas pressure $45 \mathrm{~Pa}$; shaded area — gas pressure $55 \mathrm{~Pa}$.

Fig. 8. Fluorescence photomicrographs of NIH $3 \mathrm{~T} 3$ fibroblast cells attached to the surface of the modified P3HB polymers. a) DAPI-stained, b) FITC-stained (magnification (.1000)), and c) SEM images of the fibroblasts.

Fig. 9. The vitality of the NIH $3 \mathrm{~T} 3$ cells seeded on the untreated and plasma-treated P3HB surface at days 1,3 and $7(* \mathrm{P}$ b 0.1$)$.

\section{References}

[1] M. Martina, D.W. Hutmacher, Biodegradable polymers applied in tissue engineering research: a review, Polym. Int. 56 (2007) 145-157.

[2] Guo-Qiang Chen, Qiong Wu, The application of polyhydroxyalkanoates as tissue engineering materials, Biomaterials 26 (2005) 6565-6578.

[3] K. Sudesh,H.Abe, Y. Doi, Synthesis, structure andproperties of polyhydroxyalkanoates: biological polyesters, Prog. Polym. Sci. 25 (2000) 1503-1555.

[4] V. Hasirci, Biodegradable biomedical polymers, in: D.L.Wase (Ed.), Biomaterials and Bioengineering Handbook, Marcel Dekker, New-York 2000, pp. 141-155.

[5] S. Chanprateep, Current trends in biodegradable polyhydroxyalkanoates, J. Biosci. Bioeng. 110 (2010) 621-632.

[6] G.-Q. Chen, A. Steinbuchel, Plastics completely synthesized by bacteria:polyhydroxyalkanoates, in: G.-Q. Chen (Ed.), Microbiology Monographs, SpringerVerlag, Berlin 2010, pp. 17-37.

[7] S. Lakshmi, C. Laurencin, Biodegradable polymers as biomaterials, Prog. Polym. Sci. 32 (2007) 762-798.

[8] S. Philip, T. Keshavarz, I. Roy, Polyhydroxyalkanoates: biodegradable polymers with a range of applications, J. Chem. Technol. Biotechnol. 82 (2007) 233-247.

[9] J.M. Anderson, Inflammatory response to implants, ASAIO Trans. 34 (2) (1988) 101-107.

[10] G. Altankov, K. Richau, Th. Groth, The role of surface zeta potential and substratum chemistry for regulation of dermal fibroblasts interaction,Mater.Werkst. 34 (2003) 1120-1128.

[11] K. Webb, V. Hlady, P.A. Tresco, Relative importance of surface wettability and charged functional groups on NIH 3 T3 fibroblast attachment, spreading, and cytoskeletal organization, J. Biomed. Mater. Res. 41 (1998) 422-430.

[12] S.P. Valappil, S.K. Misra, A. Boccaccini, I. Roy, Biomedical applications of polyhydroxyalkanoates, an overviewof animal testing and in vivo responses, Expert Rev. Med. Devices 3 (2006) 853-868.

[13] I. Engelberg, J. Kohn, Physico-mechanical properties of degradable polymers used in medical applications: a comparative study, Biomaterials 12 (1991) 292-304.

[14] J. Musil, Recent advances in magnetron sputtering technology, Surf. Coat. Technol. 100 (1998) 280-286.

[15] T. Pompe, K. Keller, G. Mothes, M. Nitschke, M. Teese, R. Zimmermann, C. Werner, Surface modification of poly(hydroxybutyrate) films to control cell-matrix adhesion, Biomaterials 28 (2007) 28-37. 
[16] S.A. Mirmohammadi, M.T. Khorasani, H. Mirzadeh, S. Irani, Investigation of plasma treatment on poly (3-hydroxybutyrate) film surface: characterization and invitro assay, Polym.Plast. Technol. Eng. 51 (2012) 1319-1326.

[17] I. Keen, P. Broota, L. Rintoul, P. Fredericks, M. Trau, L. Grondahl, Introducing amine functionalities on a poly(3-hydroxybutyrate-co-3-hydroxyvalerate) surface: comparing the use of ammonia plasma treatment and ethylenediamine aminolysis, Biomacromolecules 7 (2006) 427434.

[18] Y. Wang, L. Lu, Y. Zheng, X. Chen, Improvement in hydrophilicity of PHBV films by Plasma Treatment, 76AWiley Periodicals, Inc, 2005 589-595.

[19] M. Nitschke, G. Schmack, A. Janke, F. Simon, D. Pleul, C. Werner, Lowpressure plasma treatment of poly(3-hydroxybutyrate): toward tailored polymer surfaces for tissue engineering scaffolds, J. Biomed. Mater. Res. 59 (2002) 632-638.

[20] T.G. Volova, E.G. Kiselev, O.N. Vinogradova, E.D. Nikolaeva, A.A. Chistyakov, A.G. Sukovatyi, E.I. Shishatskaya, A glucose-utilizing strain, Cupriavidus eutrophus B-10646: growth kinetics, characterization and synthesis of multicomponent PHAs, PLoS One 9 (2014) 1-15.

[21] A.P. Bonartsev, V.L.Myshkina, D.A. Nikolaeva, E.K. Furina, T.A.Makhina, V.A. Livshits, A.P. Boskhomdzhiev, E.A. Ivanov, A.L. Iordanskii, G.A. Bonartseva, Biosynthesis, biodegradation, and application of poly(3-hydroxybutyrate) and its copolymers -natural polyesters produced by diazotrophic bacteria, Formatex Span 1 (2007) 295-307.

[22] T.G. Volova, Polyhydroxyalkanotes - Plastic Materials of the 21st Century: Production, Properties, Application, Nova Science Publishers, Inc, New York, 2004.

[23] T. Volova, E. Shishatskaya, V. Sevastianov, S. Efremov, O. Mogilnaya, Results of biomedical investigations of PHB and PHB/PHV fibers, Biochem. Eng. J. 16 (2003) 125-133.

[24] E.I. Shishatskaya, O.N. Voinova,A.V. Goreva, O.A. Mogilnaya, T.G. Volova, Biocompatibility of polyhydroxybutyrate microspheres: in vitro and in vivo evaluation, J. Mater. Sci. Mater. 19 (2008) 2493-2502.

[25] T. Freier, C. Kunze, C. Nischan, S. Kramer, K. Sternberg, M. Sass, U.T. Hopt, K.-P. Schmitz, In vitro and in vivo degradation studies for development of a biodegradable patch based on poly(3-hydroxybutyrate), Biomaterials 23 (2002) 2649-2657.

[26] J.M. Goddard, J.H. Hotchkiss, Polymer surface modification for the attachment of bioactive compounds, Prog. Polym. Sci. 32 (2007) 698-725.

[27] C.A. Figueroa, T. Czerwiec, C. Driemeier, I.J.R. Baumvol, S. Weber, Nitrogen diffusion enhancement in a ferrous alloy by deuterium isotopic effect, J. Appl. Phys. 101 (2007) 116106.

[28] Ahmed Mohamed El-Hadi, Influence of microcrystalline cellulose fiber (MCCF) on the morphology of poly(3-hydroxybutyrate) (PHB), Colloid Polym. Sci. 261 (2013) 743-756.

[29] Yen Leng Pak, Mansor Bin Ahmad, Kamyar Shameli, Wan.Md.Zin.Wan. Yunus, Nor Azowa Ibrahim, Norhazlin Zainuddin, Mechanical and morphological properties of poly-3hydroxybutyrate/poly(butyleneadipate-co-terephthalate)/layered double hydroxide nanocomposites, J. Nanomater. 2013 (2013) 1-8.

[30] T. Furukawa, H. Sato, R. Murakami, et al., Structure, dispersibility, and crystallinity of poly(hydroxybutyrate)/poly(L-lactic acid) blends studied by FT-IR microspectroscopy and differential scanning calorimetry, Macromolecules 38 (2005) 6445-6454.

[31] G. Kister, G. Cassanas, M. Vert, Effects of morphology, conformation and configuration on the IR and Raman spectra of various poly(lactic acid)s, Polymer 39 (1998) 267-273.

[32] Feng Shen, Erlin Zhang, ZunjieWei, Surface bio-modification of poly(hydroxybutyratecohydroxyhexanoate) and its aging effect, Colloids Surf. B: Biointerfaces 73 (2009) 302-307.

[33] A. Marmur, E. Bittoun, WhenWenzel and Cassie are right: reconciling local and global considerations, Langmuir 25 (2009) 1277-1281.

[34] L. Fernandez, M. Sanchez, F.J. Carmona, L. Palacio, J.I. Calvo, A. Hernandez, P.Pradanos, Analysis of the grafting process of PVP on a silicon surface by AFM and contact angle, Langmuir 27 (2011) 11636-11649. 
[35] L. Ponsonnet, K. Reybier, N. Jaffrezic, V. Comte, C. Lagneau, M. Lissac, C. Martelet, Relationship between surface properties (roughness, wettability) of titanium and titanium allows and cell behavior, Mater. Sci. Eng. C 23 (2003) 551-560.

[36] S.A. Redey, M. Nardin, D. Bernache-Assolant, C. Rey, P. Delannoy, L. Sedel, P.J. Marie, Behavior of human osteoblastic cells on stoichiometric hydroxyapatite and type A carbonate apatite: role of surface energy, J. Biomed. Mater. Res. 50 (2000) 353-364.

[37] F. Kok, V. Hasirci, Polyhydroxybutyrate and its copolymers: applications in the medical field, in:M.J. Yaszemski, D.J. Trantolo,K.-U. Lewandrowski,V.Hasirci,D.E.Altobelli,D.L. Wise (Eds.), Tissue Engineering and Novel Delivery Systems, Marcel Dekker, Inc., New York Basel, 2004 (620 pp.).

[38] B.D.Ulery, L.S.Nair, C.T. Laurencin, Biomedical applications of biodegradable polymers, J. Polym. Sci. B Polym. Phys. 49 (2011) 832-864.

[39] S. Cheng, G.-Q. Chen, M. Leski, B. Zou, Y. Wang, Q. Wu, The effect of D,L- $\beta$ hydroxybutyric acid on cell death and proliferation in L929 cells, Biomaterials 27 (2006) 37583765 . 